\title{
Optical Characterization of Silver Nanorod Thin Films Grown Using Oblique Angle Deposition
}

\author{
Michael Benson, ${ }^{1}$ Piyush Shah, ${ }^{2}$ Michael Marciniak, ${ }^{1}$ \\ Andrew Sarangan, ${ }^{2}$ and Augustine Urbas ${ }^{3}$ \\ ${ }^{1}$ Department of Engineering Physics, Air Force Institute of Technology, Wright-Patterson AFB, Dayton, OH 45433, USA \\ ${ }^{2}$ Electro-Optics Graduate Program, University of Dayton, Dayton, OH 45469, USA \\ ${ }^{3}$ Materials and Manufacturing Directorate, Air Force Research Laboratory, Wright-Patterson AFB, Dayton, OH 45433, USA \\ Correspondence should be addressed to Michael Benson; mrbenson403@gmail.com
}

Received 18 November 2013; Accepted 2 April 2014; Published 15 May 2014

Academic Editor: Jong Su Kim

Copyright (C) 2014 Michael Benson et al. This is an open access article distributed under the Creative Commons Attribution License, which permits unrestricted use, distribution, and reproduction in any medium, provided the original work is properly cited.

\begin{abstract}
Nanorods are metamaterial structures that have been shown to have wide application, ranging from biomedical uses to photovoltaic materials. These materials have unique optical characteristics. In this paper, two silver (Ag) nanorod thin-film samples are created using Glancing Angle Deposition (GLAD) at both near-room temperature ( $\sim 300 \mathrm{~K})$ and cryogenic temperature ( 100 K). Generalized ellipsometry is used to measure the optical constants of the samples. The strong difference between the optical constants of the constituent materials and those of these thin films shows that the characteristics of the samples are due to how their metamaterial structures are defined. The principle optical axes of the films align well with the morphological characteristics of the nanostructures. The axis with the greatest index of refraction remains aligned to the principle axes but shifts orientation with respect to morphological characteristics between samples. Experimental results show differences in both magnitude and characteristics of the nanorod indexes. Reflectance and transmittance measurements are performed to extract absorptance data. The room temperature deposited sample shows a higher overall absorptance, while the cryogenic sample shows a clear orientationdependent absorptance. Polarization data is analyzed to show that the $100 \mathrm{~K}$ thin film exhibits polarization-dependent absorptance, while the $300 \mathrm{~K}$ sample's absorptance has a strong orientation dependence.
\end{abstract}

\section{Introduction}

Nanorods can create materials with optical properties that are drastically different than their bulk material counterparts [1-3]. These structures have found a wide number of potential uses, ranging from biomedical applications [4] to photovoltaic device improvements [5]. The characterization of these structures has been studied extensively in a number of different places [6-9], focusing mostly on the optical constants of the material. Here, two nanorod samples are characterized using generalized ellipsometry and reflectancebased measurements to examine their varying optical properties, as well as their spectral absorptance properties.

\section{Materials and Methods}

2.1. Growth Methodology. The thin-film deposition method discussed in this study was done using a generic e-beam evaporation system (MDC evap-4000) made by Torr International. This chamber was retrofitted with a custom-built substrate holder to support thin-film growth at cryogenic $(\sim 100 \mathrm{~K})$ and room $(\sim 300 \mathrm{~K})$ temperatures concurrently. Liquid nitrogen was allowed to flow through the substrate holder in an open-loop configuration to achieve cryogenic substrate temperatures during the deposition process. Copper and teflon angle blocks with a preset angle of $88^{\circ}$ were mounted on the substrate holder. Using mechanical clips, silicon (Si) substrates were attached to the angled surface of the block. 
Two type-K thermocouples were attached to the angle blocks to measure substrate temperature during deposition. This experimental setup is shown in Figure 1. Further details of this experimental setup is discussed in [10].

Prime grade double-side-polished (DSP) p-type Si $\langle 100\rangle$ wafers were used in this study. Partial wafers were cleaned using acetone, methanol, and isopropyl alcohol followed by nitrogen blow drying. The silver (Ag) evaporation pellets were of $99.99 \%$ purity from Kurt J. Lesker Company. The chamber base pressure was less than $66 \times 10^{-6} \mathrm{~Pa}(0.5 \times$ $10^{-6}$ Torr) prior to the start of the deposition. After the initial preconditioning ramp, the final deposition rate was maintained at $0.3 \mathrm{~nm} / \mathrm{s}$. Film thickness was monitored using SQC-310 thin-film deposition controller.

Top view and cross sectional scanning electron microscope (SEM) images were acquired using Hitachi S-4800 high resolution SEM system and are shown in Figure 2. ImageJ software was used to structurally characterize morphological differences among the $300 \mathrm{~K}$ and $100 \mathrm{~K}$ grown Ag nanorod thin-film samples.

2.2. Spectral Ellipsometry Measurements. Ellipsometry measures the complex reflection coefficient [11]. This data is typically compared against a model of the material components to evaluate the actual parameters of the sample. In spectral ellipsometry, a broadband source is combined with a series of polarizing optics to illuminate a sample with an exact polarization state. A detector then views the specular reflectance of the sample through another polarizing optic to measure the proportion of light reflected by the sample.

The complex reflection coefficient can be described as

$$
\rho_{p p}=\tan \Psi_{p p} \exp \left[i \Delta_{p p}\right],
$$

where $\tan \left(\Psi_{p p}\right)$ is the ratio of the amplitude of light horizontally polarized over that vertically polarized, and $\Delta_{p p}$ is the phase delay between the two orthogonal fields [12].

Fundamentally, this complex reflection coefficient relates back to the reflection Jones matrix of the sample. An element with a reflection matrix of

$$
R_{j}=\left[\begin{array}{ll}
r_{p p} & r_{s p} \\
r_{p s} & r_{s s}
\end{array}\right]
$$

will have a complex reflection coefficient that is equal to $\rho_{p p}=r_{p p} / r_{s s}$. Two other complex reflection coefficients are commonly used,

$$
\begin{gathered}
\rho_{s p}=\frac{r_{s p}}{r_{s s}}, \\
\rho_{p s}=\frac{r_{p s}}{r_{p p}},
\end{gathered}
$$

and give new information on the relative Jones matrix. These reflection coefficients (and hence corresponding $\Psi$ and $\Delta$ ) can be extracted using generalized ellipsometry. Extraction methods are detailed in [13]. These additional two reflection coefficients are very important for measuring biaxial samples. A biaxial material's Jones matrix is nondiagonal everywhere

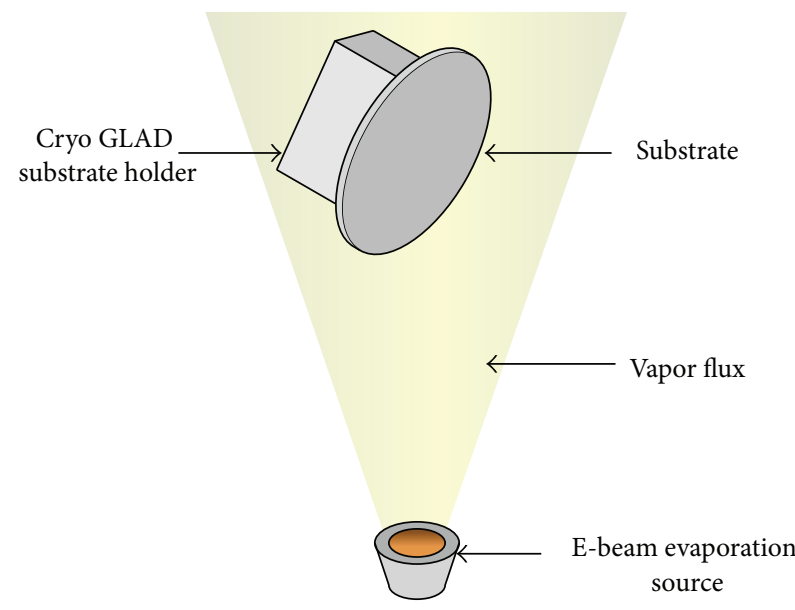

FIGURE 1: Nanorod growth experimental setup.

except for the principle axes. These extra measurements are required to properly extract the material parameters.

This data is typically measured as a function of angle of incidence, $\psi$, as measured from the surface normal. The reflection coefficient changes significantly given any change of material parameters, such as layer depth or surface roughness, making the measure of $\Psi$ and $\Delta$ over a range of angles fairly material specific.

The complex reflection coefficient, and hence $\Psi$ and $\Delta$, can easily be modeled given material parameters. Because these parameters are calculated, they can be quickly varied by a computer to create a broad range of $\Psi$ and $\Delta$ data. These simulations are then compared to the measured data. If the modeled data and the measured data align well, it can be assumed that the sample is well described by the model.

Nanorods typically exhibit biaxial properties [3, 14-16], which means that the complex refractive index, $n+i k$, is not the same for every orientation or angle $\psi$. Biaxial materials have three principle axes $(a, b, c)$, each of which has a different index of refraction. In many applications, these axes do not line up with the laboratory frame of reference, which makes them difficult to measure [15]. Ellipsometric models can be set up to generate biaxial data, which can then be used to estimate the angles of these axes. Typically, these angles correspond with properties of the materials, such as the direction of the nanorods [16].

In our experiment, we found that the axis with the highest real part of the refractive index measured at the lowest sampled wavelength changed between samples. Due to the confusion of having the axes' names changing between samples, these axes are instead designated as $\alpha, \beta$, and $\gamma$, which were chosen to correspond with the morphological characteristics of the sample and are aligned with the optical axis. $\alpha$ corresponds with the direction of the nanorods, $\gamma$ is perpendicular to $\alpha$ in the plane of the substrate, and $\beta$ is perpendicular to both $\alpha$ and $\gamma$, pointing away from the surface. In other models, $\alpha$ and $\beta$ do not have to necessarily be perpendicular to each other. Here, they are defined as perpendicular. 


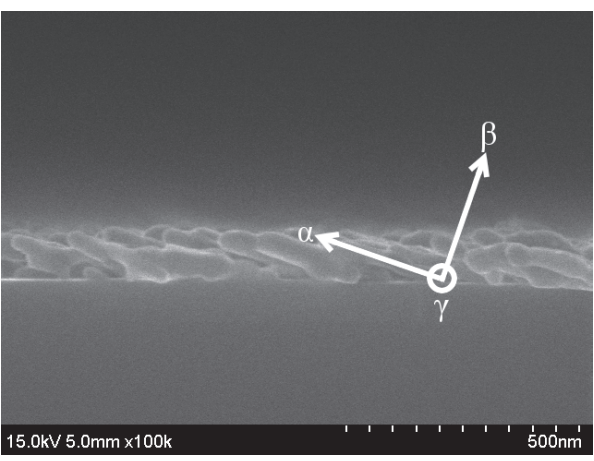

(a)

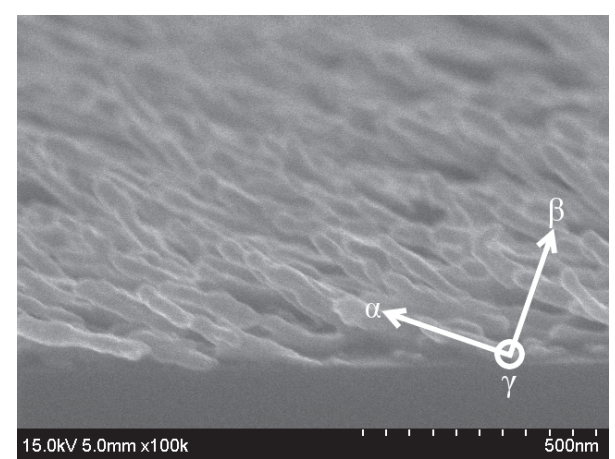

(b)

FIGURE 2: SEM images of silver nanorods. (a) Sample was grown at $300 \mathrm{~K}$, while the (b) sample was grown at $100 \mathrm{k}$.

Previous studies of nanorods point to the fact that the material will have biaxial properties, and these axes typically align with the direction of the rods [17]. Knowing this, the ellipsometric measurements were taken along three directions: the plane of $\alpha$ and $\beta$ on the side of $\alpha$ (also designated "INTO," as the nanorods point towards the source), the same plane on the opposite side (also designated "AGAINST," as the nanorods point away from the source), and the plane of the sample normal and $\gamma$ (also designated "ACROSS," as the nanorods are perpendicular to the source). This allows us to collect data along all three of the principle axes of the sample, which can be used to extract the relevant material parameters using an ellipsometric model. An example of the experimental setup used showing the sample orientation can be seen in Figure 3. Data collection was performed using J. A. Woollam Co.s IR-VASE, and analysis was carried out using the associated software [18].

\subsection{Hemispherical Reflectance and Transmittance Measure-} ments. While ellipsometry measures specularly reflected light, light scattered at other angles can also be used to identify specific material parameters, such as absorptance. Typically, absorptance is fairly difficult to measure directly, but it can be extracted from reflectance and transmittance data. Conservation of energy states that absorptance $(A)$, reflectance $(R)$, and transmittance $(T)$ are related by

$$
1=A(\psi)+R(\psi)+T(\psi) .
$$

Absorptance can also be found given the $n$ and $k$ values of the material. Absorptance is calculated as

$$
A=\exp \left[-\frac{4 \pi k}{\lambda_{0}} \frac{d}{\cos \theta}\right]
$$

where $\lambda_{0}$ is the free-space wavelength, $d$ is the material thickness, and $\theta$ is the angle of propagation within the sample. Because $k$ is divided by $\lambda_{0}$, samples will have high absorptance when they have lower $k$ (but $>0$ ) values at shorter wavelengths or higher $k$ values at longer wavelengths.

Reflectance and transmittance measurements were made using Surface Optics Corp.s SOC-100 which measures hemispherical directional reflectance (HDR) and hemispherical

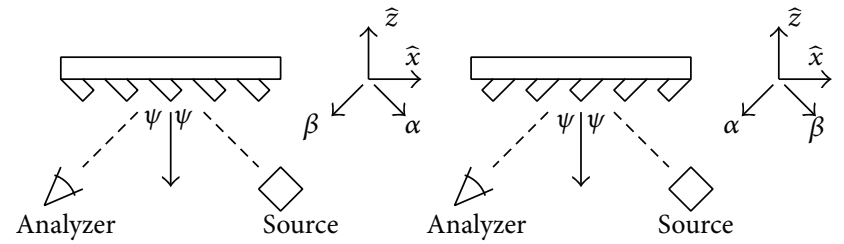

FIGURE 3: Ellipsometry experimental setup. The diagram on the left demonstrates the orientation "INTO," while the diagram on the right demonstrates the orientation "AGAINST."

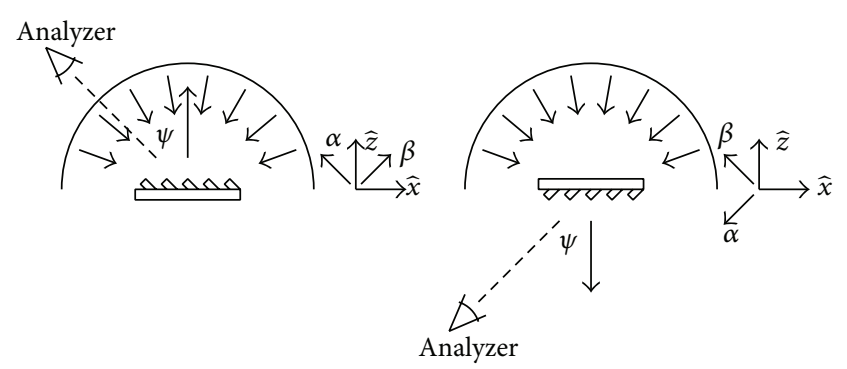

FIgURE 4: Reflectance (left) and transmittance (right) experimental setup. Both diagrams show the orientation "INTO."

directional transmittance (HDT). Because of this, special care must be taken to ensure proper measurement of these nanorod samples. The sample must be mounted upside down relative to the reflectance measurement to properly measure the transmittance of the sample. This is shown in Figure 4.

\section{Results and Discussion}

3.1. Sample Characteristics. Figure 2 showed the top and side view SEM images for $300 \mathrm{~K}$ and $100 \mathrm{~K}$ grown $\mathrm{Ag}$ nanorod thin films. The distinct morphological differences in the structure of these films are evident from these images. The nanostructure of $300 \mathrm{~K}$ grown films is in agreement with previously reported results in literature $[19,20]$. Compared to $300 \mathrm{~K}, 100 \mathrm{~K}$ grown thin films appear to have distinctly separate nanorods with smaller diameter. The $300 \mathrm{~K}$ sample has partially collapsed nanostructure with larger-diameter 

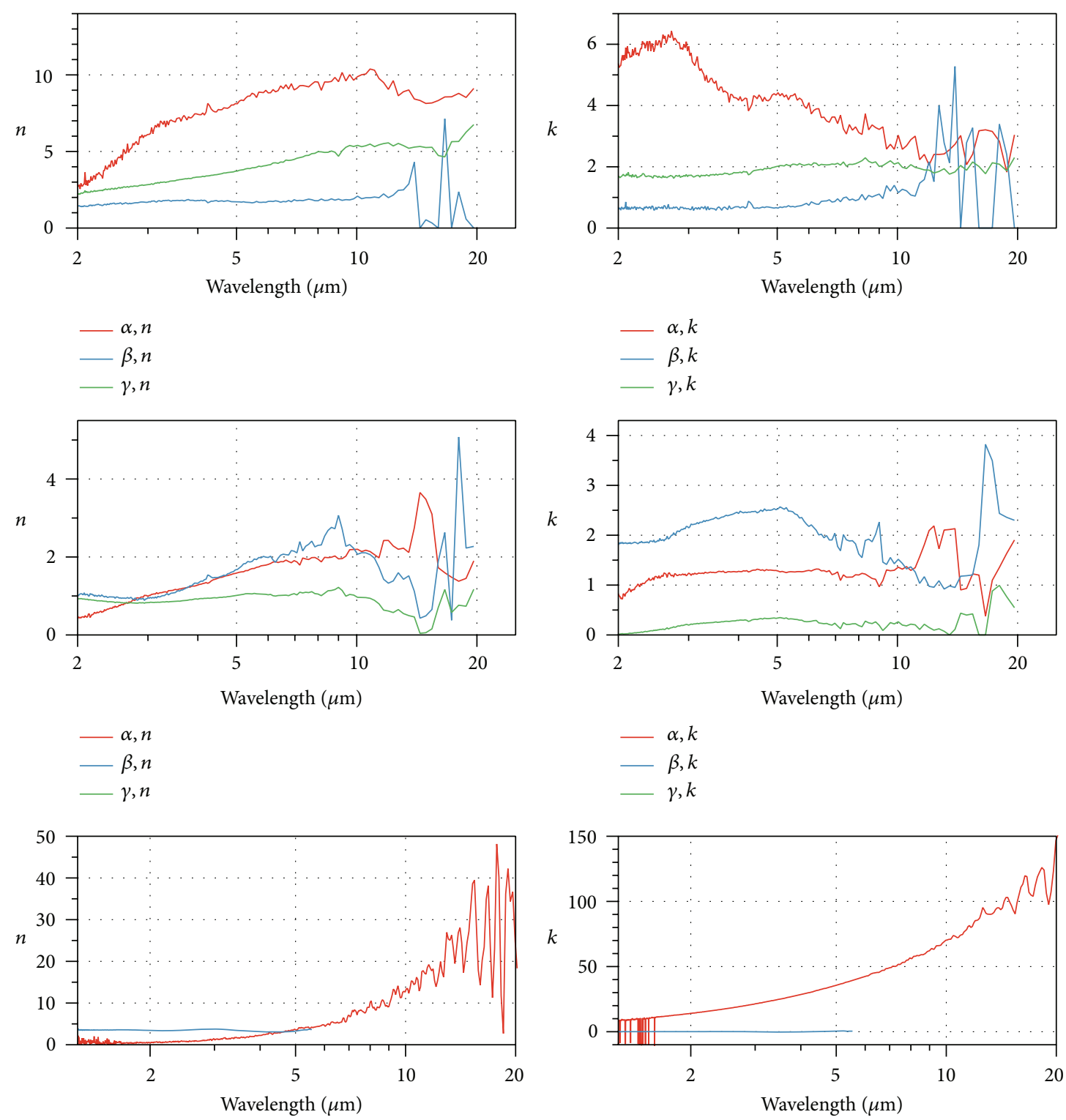

- Ag, $n$

- Ag, $k$

_ Si, $k$

(a)

(b)

FIGURE 5: Measured $n$ (a) and $k$ values (b). Sample grown at $300 \mathrm{~K}$ is shown on top, while the sample grown at $100 \mathrm{~K}$ is shown in the middle. $\alpha$ corresponds with the direction of the nanorods, $\gamma$ is the perpendicular of $\alpha$ lying in the plane of the sample, and $\beta$ is the perpendicular to the two previous vectors. The $n$ and $k$ values extracted for the Si substrate and Ag deposition are shown at the bottom.

nanorods. This fact is not obvious unless the structure of the film is investigated using cross sectional view. Based on the observed differences in the diameter, length, number of nanorods per unit area, and nanorod spacing, it appears that the available Ag nanostructured surface area of the $100 \mathrm{~K}$ films will be larger than that of the $300 \mathrm{~K}$ grown thin films. Therefore, it is expected that the $100 \mathrm{~K}$ and $300 \mathrm{~K}$ grown thin films will exhibit different plasmonic, optical, electrical, and mechanical properties. The difference in the evolution of thin films and quantitative morphological differences of these films is discussed in our previously reported study [10].

3.2. Optical Constant Extraction. The $n$ and $k$ values extracted along the principle axes are shown in Figure 5. The structure of the nanorods obviously has a strong impact on the overall characteristics of the metamaterial as a whole. Neither sample exhibits any behavior that can be attributed to a simple layered material model. These values were extracted by fitting the measured data to a biaxial layered model (summarized 
TABLE 1: Modeling parameters and measurements for both sample grown at $300 \mathrm{~K}$ and sample grown at $100 \mathrm{~K}$.

\begin{tabular}{lcccc}
\hline & \multicolumn{2}{c}{$300 \mathrm{~K}$ sample } & \multicolumn{2}{c}{$100 \mathrm{~K}$ sample } \\
& Model parameter & SEM measurement & Model parameter & SEM measurement \\
\hline Layer depth $(\mathrm{nm})$ & $135 \mathrm{~nm}$ & $128 \mathrm{~nm}-160 \mathrm{~nm}$ & $115 \mathrm{~nm}$ & $110 \mathrm{~nm}-120 \mathrm{~nm}$ \\
Nanorod angle (deg.) & $84^{\circ}$ & $68^{\circ}-72^{\circ}$ & $84^{\circ}$ & $68^{\circ}-72^{\circ}$ \\
\hline
\end{tabular}

in Table 1). Given the average film thickness (taken from the SEM images), $n$ and $k$ were varied to match the collected generalized ellipsometry data.

Both samples exhibit clear anisotropic behavior. The effective index changes considerably as a function of the orientation and the angle $\psi$. More significantly, each sample demonstrates unique behavior, indicating that the subwavelength structures are responsible for the material's response. Both sets of nanorods have complex indexes significantly higher than that of the substrate and significantly lower than that of the Ag deposition (also shown in Figure 5).

The $300 \mathrm{~K}$ sample has an index that is highest along the direction of the nanocolumns. This behavior has been observed in other nanorod samples at shorter wavelengths [9]. This index has a high complex component, which would imply that the absorptance in this direction would be comparatively higher. The complex portion decreases sharply towards $5 \mu \mathrm{m}$, where it approaches the behavior of the other two axes.

The $100 \mathrm{~K}$ sample has a much different behavior. Here, the index along the $\alpha$ axis starts lower than the other two, then increases to approximately the behavior of the $\beta$ axis. This behavior has also been observed in other nanorod samples [16]. This axis has a complex part of the index that is lower than the $\beta$ axis, which is contrary to the $300 \mathrm{~K}$ sample. The real portion of the index along $\alpha$ crosses the $\gamma$ index at $2.65 \mu \mathrm{m}$ and crosses the $\beta$ at both $2.82 \mu \mathrm{m}$ and $3.75 \mu \mathrm{m}$.

While both of these behaviors have been noted before, these samples are novel because they exhibit both behaviors. The most significant difference between the two samples is the nanostructures, implying that this difference is responsible for this unique behavior.

These two structures have significantly different effective optical constants. The optical axes of these materials always roughly align with the morphological characteristics of the sample, which is why the $\alpha, \beta$, and $\gamma$ axes were chosen instead of the typical $n_{a}, n_{b}$, and $n_{c}$ axes found in similar papers. Choosing these axes allows for a much clearer comparison between the two samples.

Similar structures have been well described using a Bruggeman effective medium approximation [6]. Typically, this kind of film would be a silver and void mixture with a void fraction percentage likely around $70 \%$ with some depolarization factor. For our particular sample, these parameters were not of great importance; the effective index of the material was needed to correlate with the measured absorptance data.

The orientation of the optical axes was easily extracted for both samples and is shown in Figure 2. For the $300 \mathrm{~K}$ sample, the orientation of the $\alpha$ axes is located at $83.76^{\circ}$ from normal. Fitting placed $\gamma$ at $89.9^{\circ}$ from normal and $\beta$ axis at $6.21^{\circ}$ from normal, on the opposite side of the $\alpha$ axis. This confirms that

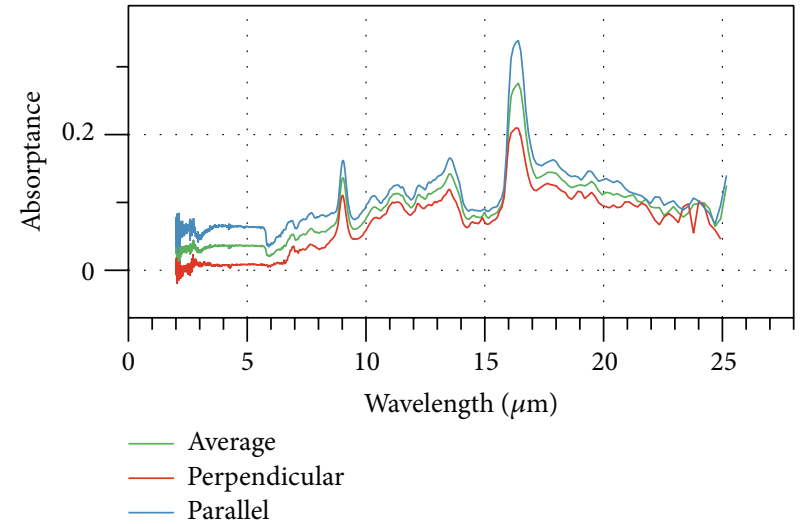

Figure 6: Polarization-based absorptance for Si substrate.

the $\gamma$ axis is in the plane of the sample. Similarly, for the $100 \mathrm{~K}$ sample, the orientation of the $\alpha$ axis was located at $83.89^{\circ}$ from normal. The $\beta$ axis is oriented at $8.26^{\circ}$ from normal. $\gamma$ axis is a bit further from the plane of the substrate, oriented $5.53^{\circ}$ above the plane of the sample.

3.3. Absorptance Calculations. The absorptance is calculated using (4) with the reflectance and transmittance measurements. The absorptance of the $\mathrm{Si}$ substrate is shown in Figure 6. The absorptance of the substrate shows that two peaks inconsistent with the ellipsometric data discussed in Section 3.2. The peaks occurring at $9 \mu \mathrm{m}$ and $16 \mu \mathrm{m}$ are due to interstitial oxygen present in the Si. This is commonly found in silicon grown by Czochralski method [21].

Note that the absorptance is polarization dependent. This is due to the difference in reflectance between the two orthogonal states; the perpendicular polarization state is prevented from exiting the material. The reflected portion of the energy passes through the layer again, where it has another chance to be absorbed, which in turn leads to a higher absorptance.

The spectral reflectance and transmittance measurements of the samples are shown in Figure 7 . The $300 \mathrm{~K}$ sample has a significantly lower reflectance than the $100 \mathrm{~K}$ sample. The "INTO" orientation reflects significantly better than the "AGAINST" orientation; the "AGAINST" and "ACROSS" transmittance measurements are nearly identical for this sample; and the "INTO" orientation transmits better at longer wavelengths.

The $100 \mathrm{~K}$ sample has clearly different reflectance and transmittance properties (Figure 7(b)). All three orientations have very similar characteristics, with the "ACROSS" orientation showing slightly higher reflectance. For transmittance, 


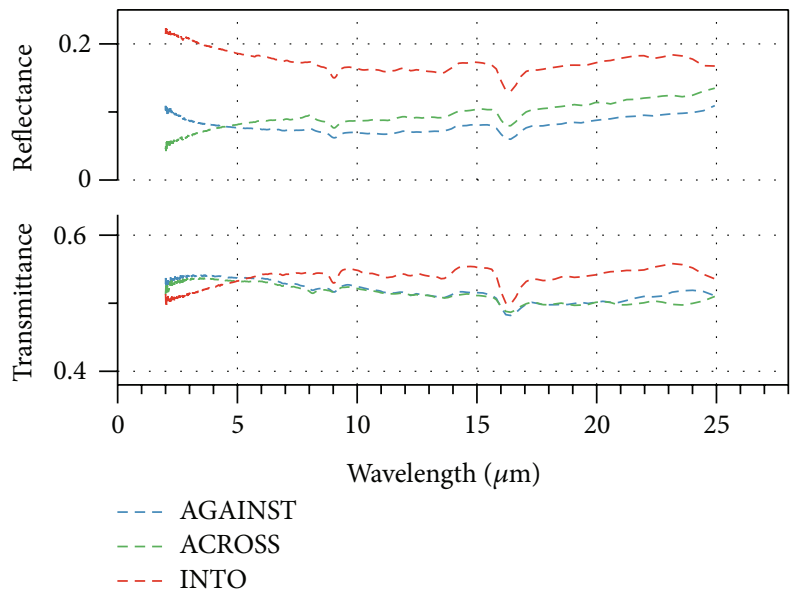

(a)

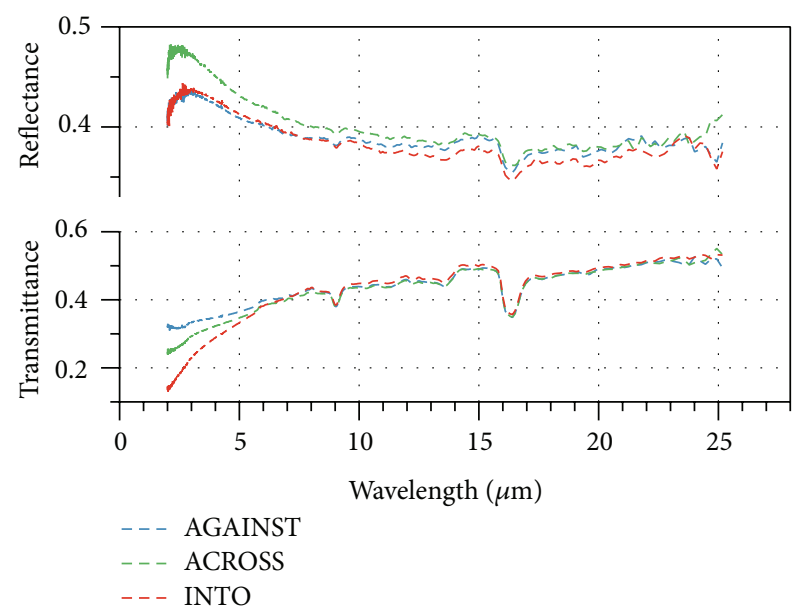

(b)

FIGURE 7: Reflectance and transmittance measurements for unpolarized light at $\psi=70^{\circ}$. (a) Sample was grown at $300 \mathrm{~K}$, while the (b) sample was grown at $100 \mathrm{~K}$.

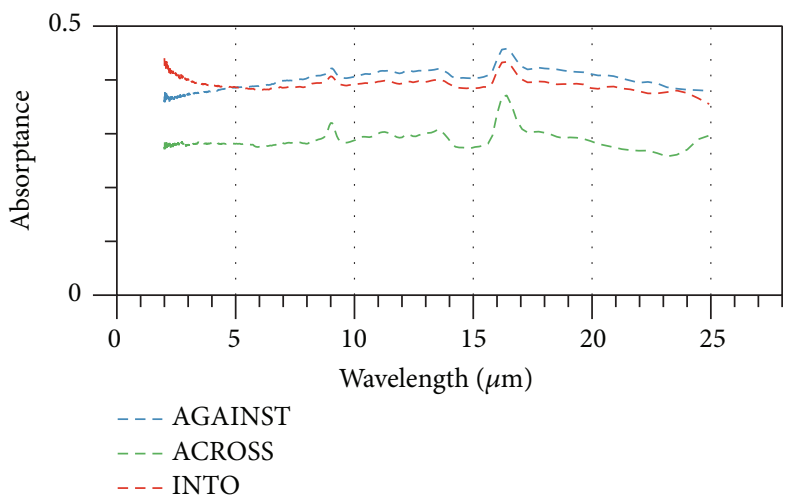

(a)

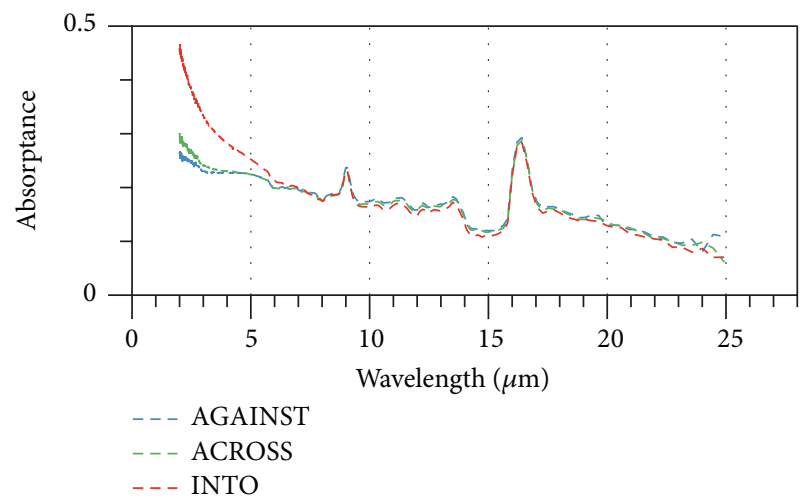

(b)

Figure 8: Absorbance measurements for unpolarized light at $\psi=70^{\circ}$. (a) Sample was grown at $300 \mathrm{~K}$, while the (b) sample was grown at $100 \mathrm{~K}$.

all three measurements are nearly identical after about $7 \mu \mathrm{m}$. For the shorter wavelengths, there is a large difference between the "AGAINST" and "INTO" orientations, contrary to what was observed in the $300 \mathrm{~K}$ sample.

The absorptance measurements show a significant difference between the two samples. These measurements are shown in Figure 8. The sample grown at $300 \mathrm{~K}$ absorbs light over the infrared wavelength range significantly better than the sample grown at $100 \mathrm{~K}$. The "INTO" absorptance is slightly higher than the "AGAINST" absorptance at wavelengths lower than about $5 \mu \mathrm{m}$. This measurement corresponds well to the indices presented in Figure 5. For example, the imaginary portion of the index along $\gamma$ is very low, which matches very well with the much lower absorptance for the "ACROSS" measurement.

For the $100 \mathrm{~K}$ sample, all three orientations have nearly identical absorptance at wavelengths longer than about $6 \mu \mathrm{m}$. At shorter wavelengths, the "INTO" orientation has a much stronger absorptance than the other two orientations. This is a characteristic shared by both samples. "ACROSS" and "AGAINST" have very similar absorptances throughout the entire range of wavelengths examined here. Again, this matches well with the indices in Figure 5. The $\alpha$ axis has a much higher imaginary index, correlating with the higher absorptance.

The absorptance measurement can also be decomposed into separate polarization states. This is shown in Figure 9. Additional measurements (not shown here) show that the differences are due to an inability for the sample to transmit the parallel (TM) polarization state. Normally, this state is easily transmitted through the sample. The inability to transmit this energy, which has already been coupled into the material, manifests as increased absorptance for this polarization state.

Furthermore, the polarization-based absorptance displays some unique characteristics. The absorptance shows a very clear polarization dependence of the $300 \mathrm{~K}$ material. In all orientations, the parallel polarization is most strongly 

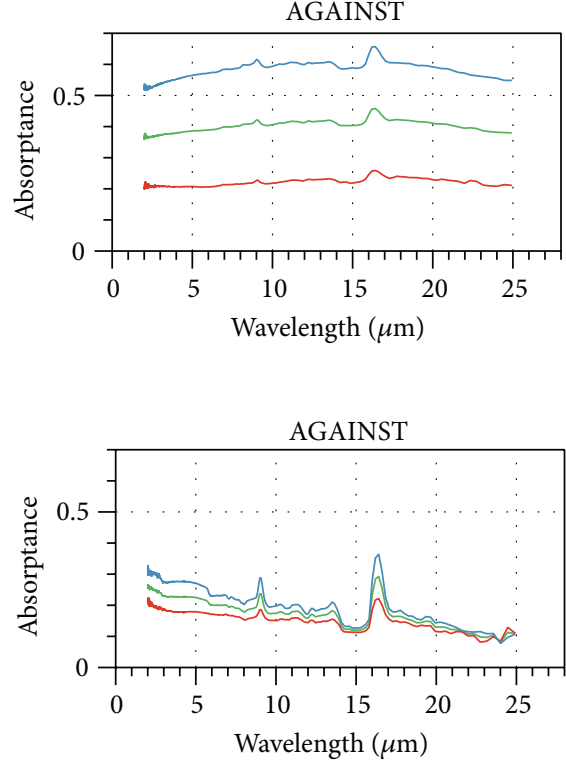

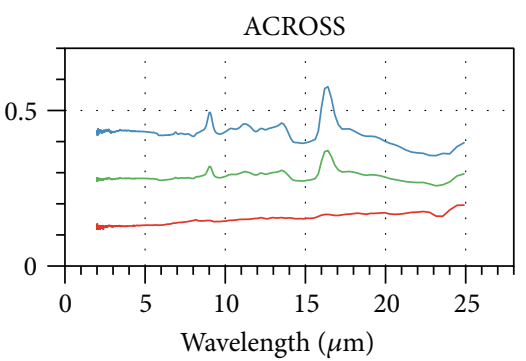

(a)

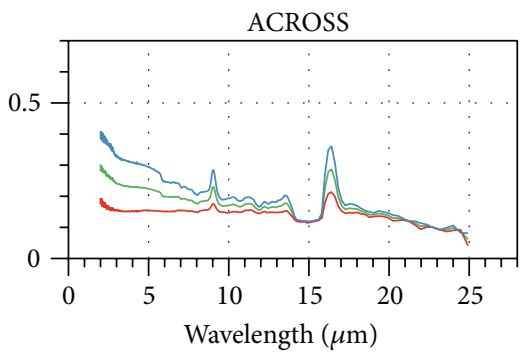

(b)
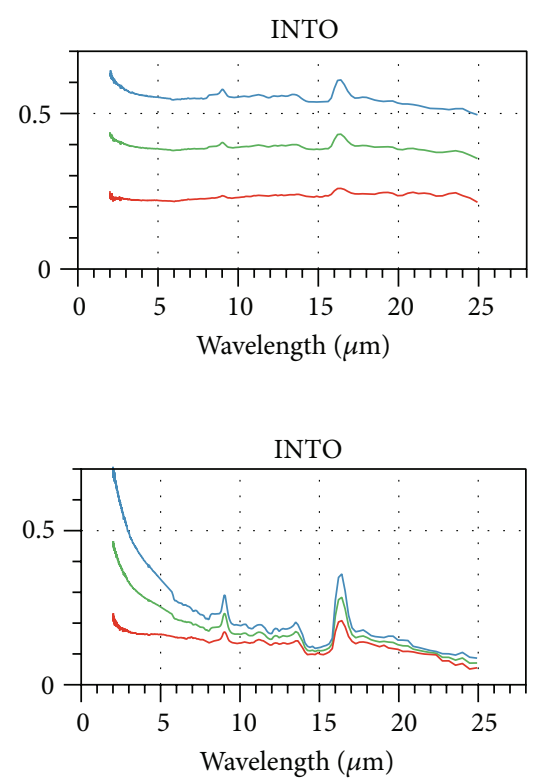

Figure 9: Polarization-based absorptance for the sample grown at $300 \mathrm{~K}$ (a) and $100 \mathrm{~K}$ (b). Blue is the parallel (TM) polarization state and red is the perpendicular (TE) polarization state. The green line is unpolarized.

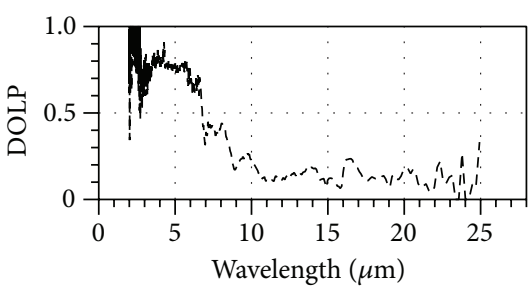

(a)

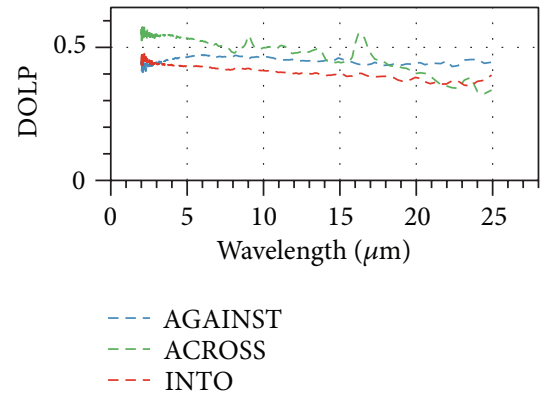

(b)

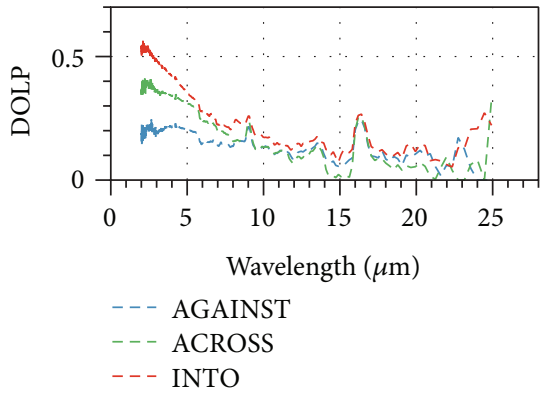

(c)

FIGURE 10: DOLP measurement for Si substrate (a), sample grown at $300 \mathrm{~K}$ (b), and sample grown at $100 \mathrm{~K}$ (c).

absorbed. The "INTO" absorptance shows the least difference between polarizations, likely due to the light being incident on the top of the nanorods. Because of the cylindrical symmetry, there is very little difference between polarization states. This would explain the lower degree of linear polarization (DOLP) for this orientation in Figure 10.

The "AGAINST" orientation shows the highest difference in absorptance between polarization states. In this case, the direction of the incident light is normal to the nanorods, which would explain why the two polarization states interact with the material so differently. "ACROSS" shows similar trends to the other two orientations, but to a lesser degree.

The polarization-based absorptance data for the sample grown at $300 \mathrm{~K}$ shows very little polarization-based absorptance (Figure 9(a)). All three orientations have similar DOLP values in Figure 10, averaging around 0.5. These values are very different than the DOLP for the Si substrate, also shown in Figure 10, which averages around 0.75 between 2 and $7 \mu \mathrm{m}$ and then drops to around 0.25 .

\section{Conclusion}

These Ag nanorod thin films have exhibited strong metamaterial properties in the sense that they are well described by an effective medium approximation. Glancing angle deposition at cryogenic temperatures created a nanorod sample that has significantly different morphological properties than a similar sample grown at room temperature. Ellipsometry reveals that these two samples have greatly varying optical properties. Notably, the optical axis with the highest refractive index changed places with respect to the axes defined by the morphological features of the nanorods between the samples. The $300 \mathrm{~K}$ sample demonstrates a much higher set of indices than does the $100 \mathrm{~K}$ sample. The $100 \mathrm{~K}$ has significantly 
different behavior than that of the $300 \mathrm{~K}$ sample. The absorptance of both samples has been measured. Absorptance measurements correspond well with the measured material indices. The $100 \mathrm{~K}$ sample exhibits a much lower difference in absorptance at wavelengths longer than $6 \mu \mathrm{m}$, while showing its absorptance is strongly orientation dependent in the shorter wavelengths. The $300 \mathrm{~K}$ sample shows a smaller orientation dependence, but across the entire infrared band. These measurements show the impact of the subwavelength structures on the observable optical properties of nanorod metamaterials.

\section{Conflict of Interests}

The authors declare that there is no conflict of interests regarding the publication of this paper.

\section{References}

[1] S.-H. Hsu, E.-S. Liu, Y.-C. Chang et al., "Characterization of Si nanorods by spectroscopic ellipsometry with efficient theoretical modeling," Physica Status Solidi A: Applications and Materials Science, vol. 205, no. 4, pp. 876-879, 2008.

[2] G. Beydaghyan, C. Buzea, Y. Cui, C. Elliott, and K. Robbie, "Ex situ ellipsometric investigation of nanocolumns inclination angle of obliquely evaporated silicon thin films," Applied Physics Letters, vol. 87, no. 15, Article ID 153103, pp. 1-3, 2005.

[3] D. Schmidt, B. Booso, T. Hofmann, E. Schubert, A. Sarangan, and M. Schubert, "Monoclinic optical constants, birefringence, and dichroism of slanted titanium nanocolumns determined by generalized ellipsometry," Applied Physics Letters, vol. 94, no. 1, Article ID 011914, 2009.

[4] A. V. Kabashin, P. Evans, S. Pastkovsky et al., "Plasmonic nanorod metamaterials for biosensing," Nature Materials, vol. 8, no. 11, pp. 867-871, 2009.

[5] W. U. Huynh, J. J. Dittmer, and A. P. Alivisatos, "Hybrid nanorod-polymer solar cells," Science, vol. 295, no. 5564, pp. 2425-2427, 2002.

[6] D. Schmidt, "Characterization of highly anisotropic threedimensionally nanostructured surfaces," Thin Solid Films, 2013.

[7] D. Liang, D. Schmidt, H. Wang, E. Schubert, and M. Schubert, "Generalized ellipsometry effective medium approximation analysis approach for porous slanted columnar thin films infiltrated with polymer," Applied Physics Letters, vol. 103, no. 11, pp. 111906-111906, 2013.

[8] K. B. Rodenhausen, D. Schmidt, T. Kasputis, A. K. Pannier, E. Schubert, and M. Schubert, "Generalized ellipsometry in-situ quantification of organic adsorbate attachment within slanted columnar thin films," Optics Express, vol. 20, no. 5, pp. 54195428, 2012.

[9] D. Schmidt and M. Schubert, "Anisotropic bruggeman effective medium approaches for slanted columnar thin films," Journal of Applied Physics, vol. 114, no. 8, Article ID 083510, 2013.

[10] P. J. Shah, X. Niu, and A. M. Sarangan, "High aspect ratio silver nanorod thin films grown at cryogenic substrate temperature," Journal of Nanoscience Letters, vol. 3, article 19, 2013.

[11] J. A. Woollam, B. D. Johs, C. M. Herzinger, J. N. Hilfiker, R. A. Synowicki, and C. L. Bungay, "Overview of variable-angle spectroscopic ellipsometry (vase): I. basic theory and typical applications," in Optical Metrology, vol. 1 of Proceedings of SPIE, pp. 3-28, 1999.
[12] D. H. Goldstein, Polarized Light, Revised and Expanded, vol. 83, CRC Press, 2003.

[13] M. Schubert, B. Rheinländer, J. A. Woollam, B. Johs, and C. M. Herzinger, "Extension of rotating-analyzer ellipsometry to generalized ellipsometry: Determination of the dielectric function tensor from uniaxial $\mathrm{TiO}_{2}$," Journal of the Optical Society of America A: Optics and Image Science, and Vision, vol. 13, no. 4, pp. 875-883, 1996.

[14] J. F. Elman, J. Greener, C. M. Herzinger, and B. Johs, "Characterization of biaxially-stretched plastic films by generalized ellipsometry," Thin Solid Films, vol. 313-314, pp. 814-818, 1998.

[15] M. Schubert and W. Dollase, "Generalized ellipsometry for biaxial absorbing materials: Determination of crystal orientation and optical constants of Sb2S3," Optics Letters, vol. 27, no. 23, pp. 2073-2075, 2002.

[16] D. Schmidt, A. C. Kjerstad, T. Hofmann, R. Skomski, E. Schubert, and M. Schubert, "Optical, structural, and magnetic properties of cobalt nanostructure thin films," Journal of Applied Physics, vol. 105, no. 11, Article ID 113508, 2009.

[17] D. Schmidt, B. Booso, T. Hofmann, E. Schubert, A. Sarangan, and M. Schubert, "Generalized ellipsometry for monoclinic absorbing materials: determination of optical constants of $\mathrm{Cr}$ columnar thin films," Optics Letters, vol. 34, no. 7, pp. 992-994, 2009.

[18] J. A. Woollam, Ellipsometry, Variable Angle Spectroscopic, Wiley Encyclopedia of Electrical and Electronics Engineering, 2000.

[19] Y.-J. Jen, A. Lakhtakia, C.-W. Yu, and C.-T. Lin, "Vapordeposited thin films with negative real refractive index in the visible regime," Optics Express, vol. 17, no. 10, pp. 7784-7789, 2009.

[20] K. R. Beavers, N. E. Marotta, and L. A. Bottomley, "Thermal stability of silver nanorod arrays," Chemistry of Materials, vol. 22, no. 7, pp. 2184-2189, 2010.

[21] Interstitial Oxygen, Sunedison, 2013. 

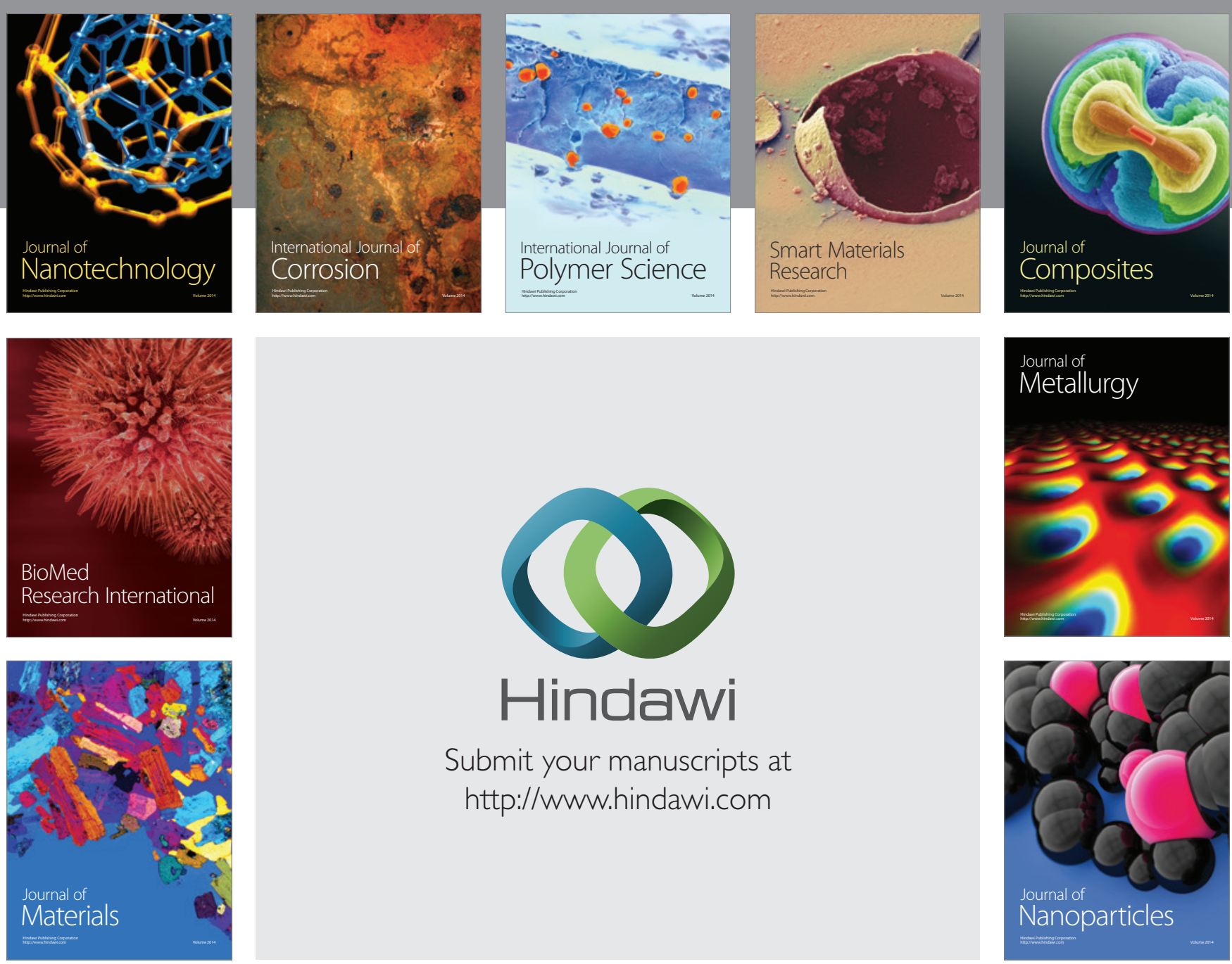

Submit your manuscripts at http://www.hindawi.com
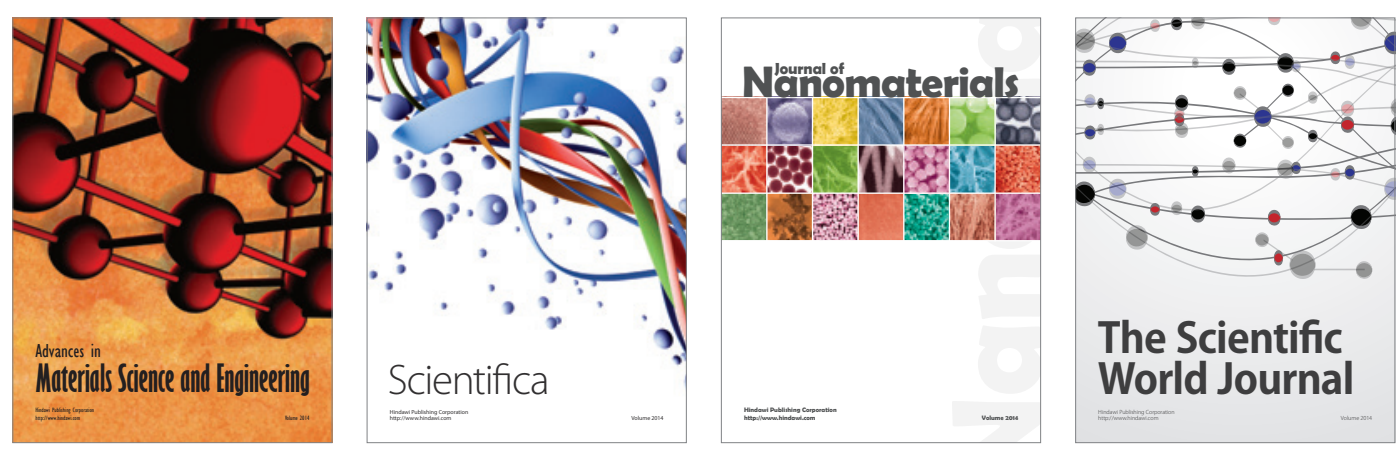

\section{The Scientific World Journal}
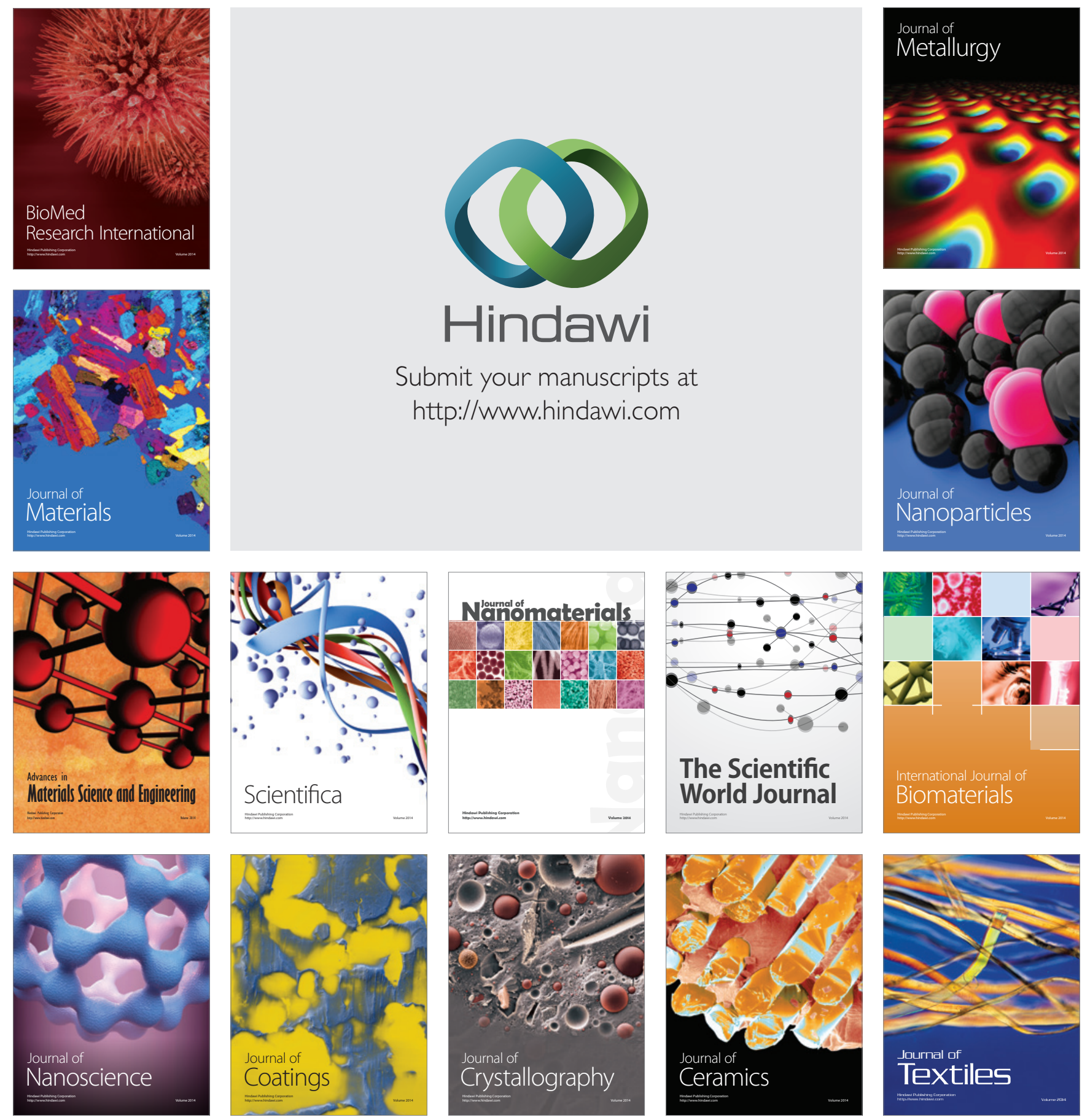